Pacific Journal of Mathematics

SOME REMARKS ON HIGH ORDER DERIVATIONS 


\section{SOME REMARKS ON HIGH ORDER DERIVATIONS}

\section{YASUNORI ISHIBASHI}

Let $k, A$ and $B$ be commutative rings such that $A$ and $B$ are $k$-algebras. In this paper it is shown that $\Omega_{k}^{(q)}\left(A \otimes_{k} B\right)$, the module of high order differentials of $A \otimes_{k} B$ can be expressed by making use of $\Omega_{k}^{(i)}(A)$ and $\Omega_{k}^{(j)}(B)$. On the other hand let $K / k$ be a finite purely inseparable field extension. Sandra Z. Keith has given a criterion for a $k$-linear mapping of $K$ into itself to be a high order derivation of $K / k$. The representation of $\Omega_{k}^{(q)}\left(A \otimes_{k} B\right)$ is used to show that Keith's result is valid for larger class of algebras.

Let $k, A$ and $B$ be commutative rings with identities such that $A$ and $B$ are $k$-algebras. $A \bigotimes_{k} B$ is an $A$-algebra (resp. a $B$-algebra) via the natural homomorphism $f_{A}$ (resp. $f_{B}$ ) such that $f_{A}(a)=a \otimes 1$ (resp. $\left.f_{B}(b)=1 \otimes b\right)$. In [5] Y. Nakai proved that there exists a direct sum decomposition

$$
\Omega_{k}^{(q)}\left(A \bigotimes_{k} B\right)=\Omega_{k}^{(q)}(A) \bigotimes_{k} B \oplus A \bigotimes_{k} \Omega_{k}^{(q)}(B) \oplus U_{A \otimes B \mid k}^{(q)} .
$$

The submodule $U_{A \otimes B \mid k}^{(q)}$ has the universal mapping property with respect to $q$ th order derivations of $A \otimes_{k} B$ which vanish on $f_{A}(A)$ and $f_{B}(B)$. In this paper we shall investigate the structure of $U_{A \otimes B \mid k}^{(q)}$. In fact we can express $U_{A \otimes B ! k}^{(q)}$ by making use of $\Omega_{k}^{(i)}(A)$ and $\Omega_{k}^{(\jmath)}(B)$ when $k$ is a field.

On the other hand Sandra Z. Keith proved

Theorem ([4]). Let $K / k$ be a finite purely inseparable field extension and let $\varphi$ be a k-linear mapping of $K$ into itself. Then we have $\varphi \in D_{0}^{(q)}(K / k)$ if and only if $\delta \varphi \in D_{0}^{(1)}(K / k)-D_{0}^{(q-1)}(K / k)+$ $D_{0}^{(2)}(K / k) \smile D_{0}^{(q-2)}(K / k)+\cdots+D_{0}^{(q-1)}(K / k) \smile D_{0}^{(1)}(K / k)$, where $\delta$ is the Hochschild coboundary operator (cf. [2]) and denotes the cupproduct.

This gives an alternative inductive definition of $q$ th order derivations which is meaningful for not-necessarily commutative rings but which possibly differs from Nakai's for commutative rings in general. In this paper we shall use our representation of $U_{A \otimes B \mid c}^{(q)}$ to show that Keith's result is generalized to larger class of algebras.

Any ring in this paper is assumed to be commutative and contain 1. Let $k$ and $A$ be commutative rings. We say that $A$ is a $k$-algebra if there exists a ring homomorphism $f$ such that $f(1)=1$. The readers are expected to refer the paper [5] for notations and terminologies. 
The author wishes to express his thanks to Professor Y. Nakai for his suggestions and encouragement.

1. Representation of $U_{A \otimes B \mid k}^{(q)}$. Let $k, A$ and $B$ be rings such that $A$ and $B$ are $k$-algebras.

Lemma 1. Let $D$ be an $m$ th order derivation of $A / k$ into an $A$-module $M$ and let $\Delta$ be an $n$th order derivation of $B / k$ into $a B$ module $N$. Then $D \otimes \Delta$ is an $(m+n)$ th order derivation of $A \otimes_{k}$ $B$ into $M \otimes_{k} N$.

Proof. We consider the idealizations $A \oplus M$ and $B \oplus N$ of $M$ and $N$ respectively. Then $D$ (resp. $\Delta$ ) is regarded as an $m$ th (resp. $n$ th) order derivation of $A$ (resp. $B$ ) into $A \oplus M$ (resp. $B \oplus N$ ). The mapping $D \otimes \Delta$ of $A \otimes_{k} B$ into $(A \oplus M) \bigotimes_{k}(B \oplus N)$ is decomposed as follows:

$$
A \otimes B_{k} \stackrel{D \otimes 1_{B}}{\longrightarrow}(A \oplus M) \bigotimes_{k} B \stackrel{1_{A \otimes M} \otimes \Delta}{\longrightarrow}(A \oplus M) \bigotimes_{k}(B \oplus N) .
$$

By Corollary 6.1 in [5], $D \otimes \Delta$ is an $(m+n)$ th order derivation.

The following lemmas are immediate.

Lemma 2. In $A \otimes_{k} A$ we have

$$
\begin{aligned}
(1 \otimes & \left.a_{1}-a_{1} \otimes 1\right) \cdots\left(1 \otimes a_{q}-a_{q} \otimes 1\right) \\
= & \left(1 \otimes a_{1} \cdots a_{q}-a_{1} \cdots a_{q} \otimes 1\right) \\
& +\sum_{s=1}^{q-1}(-1)^{s} \sum_{i_{1}<\cdots<i_{s}} a_{i_{1}} \cdots a_{i_{s}}\left(1 \otimes a_{1} \cdots \widehat{a}_{i_{1}} \cdots \widehat{a}_{i_{s}} \cdots a_{q}\right. \\
& \left.-a_{1} \cdots \hat{a}_{i_{1}} \cdots \widehat{a}_{i_{s}} \cdots a_{q} \otimes 1\right) .
\end{aligned}
$$

Lemma 3. Let $D$ be a $q$ th order derivation of $A \bigotimes_{k} B$ into an $A \bigotimes_{k} B$-module $M$ vanishing on $f_{A}(A)$ and $f_{B}(B)$, where $f_{A}$ (resp. $f_{B}$ ) $i$ s the homomorphism of $A$ (resp. $B$ ) into $A \otimes_{k} B$ such that $f_{A}(\alpha)=$ $a \otimes 1$ (resp. $\left.f_{B}(b)=1 \otimes b\right)$. Then we have

$$
\begin{aligned}
& D\left(a_{1} \cdots a_{i} \otimes b_{1} \cdots b_{q+1-i}\right) \\
& =\sum_{s=1}^{i-1}(-1)^{s-1} \sum_{\alpha_{1}<\cdots<\alpha_{s}}\left(a_{\alpha_{1}} \cdots a_{\alpha_{s}} \otimes 1\right) \\
& \times D\left(a_{1} \cdots \hat{a}_{\alpha_{1}} \cdots \hat{a}_{\alpha_{s}} \cdots a_{i} \otimes b_{1} \cdots b_{q+1-i}\right) \\
& +\sum_{t=1}^{q-i}(-1)^{t-1} \sum_{\beta_{1}<\cdots<\beta_{t}}\left(1 \otimes b_{\beta_{1}} \cdots b_{\beta_{t}}\right) \\
& \times D\left(a_{1} \cdots a_{i} \otimes b_{1} \cdots \hat{b}_{\beta_{1}} \cdots \hat{b}_{\beta_{t}} \cdots b_{q+1-2}\right) \\
& +\sum_{s, t=1}^{s \leqq i-1, t \leqq q-i}(-1)^{s+t-1} \sum_{\substack{\alpha_{1}<\cdots<<\alpha_{s} \\
\beta_{1}<\cdots<\beta_{t}}}\left(a_{\alpha_{1}} \cdots a_{\alpha_{s}} \otimes b_{\beta_{1}} \cdots b_{\beta_{t}}\right) \\
& \times D\left(a_{1} \cdots \hat{a}_{\alpha_{1}} \cdots \hat{a}_{\alpha_{s}} \cdots a_{i} \otimes b_{1} \cdots \hat{b}_{\beta_{1}} \cdots \hat{b}_{\beta_{t}} \cdots b_{q+1-i}\right) \text {. }
\end{aligned}
$$


We denote by $\delta_{A \mid k}^{(q)}$ the canonical $q$ th order derivation of $A$ into $\Omega_{k}^{(q)}(A)$. Unless any confusion arises, $\delta_{A \mid k}^{(q)}$ is denoted by $\delta_{A}^{(q)}$ or $\delta^{(q)}$ simply. If $i \leqq j$, we have the canonical epimorphism $\varphi_{i j}$ of $\Omega_{k}^{(j)}(A)$ onto $\Omega_{k}^{(i)}(A)$ given by $\varphi_{i j}\left(\delta^{(j)} a\right)=\delta^{(i)} a$. Let $\psi_{i j}$ be the homomorphism of $\Omega_{k}^{(j)}(B)$ onto $\Omega_{k}^{(i)}(B)$ defined as above. We define the homomorphism $\Phi_{q}$ of $\bigoplus_{i=1}^{q-1} \Omega_{k}^{(i)}(A) \boldsymbol{\bigotimes}_{k} \Omega_{k}^{(q-i)}(B)$ into $\bigoplus_{i=1}^{q-2} \Omega_{k}^{(i)}(A) \boldsymbol{\otimes}_{k} \Omega_{k}^{(q-1-i)}(B)$ as follows: for $x \otimes y \in \Omega_{k}^{(i)}(A) \bigotimes_{k} \Omega_{k}^{(j)}(B)$,

$$
\Phi_{q}(x \otimes y)= \begin{cases}\varphi_{q-2, q-1}(x) \otimes y & \text { if } i=q-1, j=1 \\ \varphi_{i-1, i}(x) \otimes y-x \otimes \psi_{j-1, j}(y) & \text { if } i, j>1 \\ -x \otimes \psi_{q-2, q-1}(y) & \text { if } i=1, j=q-1 .\end{cases}
$$

Obviously $\Phi_{q}$ is surjective.

THEOREM 1. There exists a natural isomorphism

(1) $U_{A \otimes B \mid k}^{(2)} \cong \operatorname{Ker} \Phi_{2}=\Omega_{k}^{(1)}(A) \bigotimes_{k} \Omega_{k}^{(1)}(B)$,

(2) for $q \geqq 3, U_{A \otimes B \mid k}^{(q)} \cong \operatorname{Ker} \Phi_{q}$ if $k$ is a field.

Proof. We consider the mapping $\delta$ of $A \bigotimes_{k} B$ into $\bigoplus_{i=1}^{q-1} \Omega_{k}^{(i)}(A) \bigotimes_{k}$ $\Omega_{k}^{(q-i)}(B)$ defined by

$$
\delta(a \otimes b)=\sum_{i=1}^{q-1} \delta_{A}^{(i)} a \otimes \delta_{B}^{(q-i)} b .
$$

By Lemma 1 we see that $\delta$ is a $q$ th order derivation. Since the image of $\delta$ is contained in $\operatorname{Ker} \Phi_{q}, \delta$ induces a $q$ th order derivation of $A \otimes_{k} B$ into $\operatorname{Ker} \Phi_{q}$. The induced one is also denoted by $\delta$. Clearly $\delta$ vanishes on $f_{A}(A)$ and $f_{B}(B)$. We have only to prove that the pair $\left\{\operatorname{Ker} \Phi_{q}, \delta\right\}$ satisfies the universal mapping property with respect to $q$ th order derivations of $A \otimes_{k} B$ which vanish on $f_{A}(A)$ and $f_{B}(B)([5])$. Let $I_{A}$ (resp. $I_{B}$ ) be the kernel of the contraction mapping: $A \bigotimes_{k} A \rightarrow A$ (resp. $B \bigotimes_{k} B \rightarrow B$ ). We regard $I_{A} \bigotimes_{k} I_{B}$ as an $A \otimes_{k} B$-module via

$$
(a \otimes b)\{(x \otimes y) \otimes(u \otimes v)\}=(a x \otimes y) \otimes(b u \otimes v) .
$$

Under our assumption it will be shown that we have a natural isomorphism of $A \bigotimes_{k} B$-modules

$$
\operatorname{Ker} \Phi_{q} \cong I_{A} \bigotimes_{k} I_{B} / \sum_{i=1}^{q} I_{A}^{i} \otimes I_{B}^{q+1-i},
$$

where $I_{A}^{i} \otimes I_{B}^{j}$ denotes the image of the canonical homomorphism of $I_{A}^{i} \bigotimes_{k} I_{B}^{j}$ into $I_{A} \bigotimes_{k} I_{B}$. For $q=2$ our assertion is obvious. For $q \geqq$ 3 we assume that $k$ is a field. We define the $A \boldsymbol{\otimes}_{k} B$-linear mapping $\Psi$ of $I_{A} \bigotimes_{k} I_{B}$ into $\bigoplus_{i=1}^{q-1} \Omega_{k}^{(i)}(A) \bigotimes_{k} \Omega_{k}^{(q-i)}(B)$ by 


$$
\Psi((1 \otimes a-a \otimes 1) \otimes(1 \otimes b-b \otimes 1))=\sum_{i=1}^{q-1} \delta_{A}^{(i)} a \otimes \delta_{B}^{(q-i)} b .
$$

Obviously we have $\operatorname{Im} \Psi \subset \operatorname{Ker} \Phi_{q}$. We shall show that $\Psi$ is an epimorphism of $I_{A} \otimes_{k} I_{B}$ onto $\operatorname{Ker} \Phi_{q}$ with kernel $\sum_{i=1}^{q} I_{A}^{i} \otimes I_{B}^{q+1-i}$. Let $f \in I_{A} \otimes_{k} I_{B}$ and let $\pi_{i}(f)$ denote the canonical image of $f$ in $\Omega_{k}^{(i)}(A) \bigotimes_{k}$ $\Omega_{k}^{(q-i)}(B)$. We assume that $\sum_{i=1}^{q-1} \pi_{i}\left(f_{i}\right) \in \operatorname{Ker} \Phi_{q}$ for $f_{i} \in I_{A} \otimes_{k} I_{B}(1 \leqq$ $i \leqq q-1)$. From the definition of $\Phi_{q}$ we see that $f_{i}-f_{i+1} \in I_{A}^{i+1} \otimes$ $I_{B}+I_{A} \otimes I_{B}^{q-i}(1 \leqq i \leqq q-2)$. Hence we have $f_{i}+\alpha_{i}=f_{i+1}+\beta_{i+1}$ for some $\alpha_{i} \in I_{A}^{i+1} \otimes I_{B}$ and $\beta_{i+1} \in I_{A} \otimes I_{B}^{q-i}(1 \leqq i \leqq q-2)$, and so it follows that $f_{1}+\alpha_{1}+\cdots+\alpha_{q-2}=f_{2}+\beta_{2}+\alpha_{2}+\cdots+\alpha_{q-2}=\cdots=$ $f_{q-1}+\beta_{2}+\cdots+\beta_{q-1}$. Let $f$ be this equal element of $I_{A} \boldsymbol{\otimes}_{k} I_{B}$. Then we have $\pi_{i}(f)=\pi_{i}\left(f_{i}\right)$ and therefore $\Psi$ is surjective. Next we prove $\operatorname{Ker} \Psi=\sum_{i=1}^{q} I_{A}^{i} \otimes I_{B}^{q+1-i}$. Let us consider an element $g$ of $I_{A} \bigotimes_{k} I_{B}$. If $g$ is in $\operatorname{Ker} \Psi$, we have $g \in I_{A}^{i+1} \otimes I_{B}+I_{A} \otimes I_{B}^{q+1-i}(1 \leqq$ $i \leqq q-1)$ and so $g=\varepsilon_{i}+\zeta_{i}$ for suitable $\varepsilon_{i} \in I_{A}^{i+1} \otimes I_{B}$ and $\zeta_{i} \in I_{A} \otimes$ $I_{B}^{q+1-i}$. On the other hand we get $\varepsilon_{i}-\varepsilon_{i+1}=\zeta_{i+1}-\zeta_{i} \in\left(I_{A}^{i+1} \otimes I_{B}\right) \cap$ $\left(I_{A} \otimes I_{B}^{q-i}\right)=I_{A}^{i+1} \otimes I_{B}^{q-i}$ since $k$ is a field. This implies easily $g \in$ $\sum_{i=1}^{q} I_{A}^{i} \otimes I_{B}^{q+1-i}$. We wish to show that the pair $\left\{\operatorname{Ker} \Phi_{q}, \delta\right\}$ has the universal mapping property. Let $D$ be a $q$ th order derivation of $A \bigotimes_{k} B$ into an $A \bigotimes_{k} B$-module $M$ vanishing on $f_{A}(A)$ and $f_{B}(B)$. Then it suffices to prove that there is an $A \otimes_{k} B$-homomorphism $\Theta$ of $I_{A} \otimes_{k} I_{B} / \sum_{i=1}^{q} I_{A}^{i} \otimes I_{B}^{q+1-i}$ into $M$ satisfying

$$
\Theta(\pi\{(1 \otimes a-a \otimes 1) \otimes(1 \otimes b-b \otimes 1)\})=D(a \otimes b),
$$

where $\pi$ is the canonical homomorphism of $I_{A} \otimes_{k} I_{B}$ onto $I_{A} \bigotimes_{k}$ $I_{B} / \sum_{i=1}^{q} I_{A}^{i} \otimes I_{B}^{q+1-i}$. We consider the mapping $\Lambda$ of $\left(A \bigotimes_{k} A\right) \bigotimes_{k}\left(B \bigotimes_{k}\right.$ $B$ ) into $M$ defined by

$$
\Lambda((x \otimes y) \otimes(u \otimes v))=(x \otimes u) D(y \otimes v) .
$$

Since $D$ vanishes on $f_{A}(A)$ and $f_{B}(B), \Lambda$ induces the mapping of $I_{A} \otimes_{k} I_{B}$ into $M$ sending $(1 \otimes a-a \otimes 1) \otimes(1 \otimes b-b \otimes 1)$ to $D(a \otimes b)$. Now it follows from Lemmas 2 and 3 that $\Lambda$ vanishes on $\sum_{i=1}^{q} I_{A}^{i} \otimes I_{B}^{q+1-i}$, and so $A$ induces the desired mapping $\Theta$. This completes our proof.

REMARK. If $\Omega_{k}^{(i)}(A)=I_{A} / I_{A}^{i+1}$ (resp. $\left.\Omega_{k}^{(i)}(B)=I_{B} / I_{B}^{i+1}\right)$ is $k$-flat for every $i$, we have $\left(I_{A}^{i+1} \otimes I_{B}\right) \cap\left(I_{A} \otimes I_{B}^{q-i}\right)=I_{A}^{i+1} \otimes I_{B}^{q-i}$ by $[1]\left(\S 1, \mathrm{n}^{\circ} 6\right.$, Proposition 7). In this case our proof shows that we have $U_{A \otimes B \mid k}^{(q)} \cong$ $\operatorname{Ker} \Phi_{q}$ for $q \geqq 3$.

2. A generalization of the result due to Keith. Let $k$ and $A$ be rings such that $A$ is a $k$-algebra. Let $M$ and $N$ be $A$-modules. We consider the homomorphism $\omega$ of $\operatorname{Hom}_{A}(M, A) \bigotimes_{k} \operatorname{Hom}_{A}(N, A)$ into $\operatorname{Hom}_{A \otimes_{k} A}\left(M \otimes_{k} N, A\right)$ given by 


$$
[\omega(f \otimes g)](m \otimes n)=f(m) g(n)
$$

for $f \in \operatorname{Hom}_{A}(M, A), g \in \operatorname{Hom}_{A}(N, A), m \in M$ and $n \in N$. Now $A$ is regarded as an $A \bigotimes_{k} A$-module via the contraction mapping: $A \otimes_{k}$ $A \rightarrow A$.

Lemma 4. If $M$ is a finite projective A-module, then $\omega$ is an epimorphism.

Proof. When $M$ is a finite free $A$-module, our assertion is obvious. If $M$ is finite $A$-projective, $M$ is a direct summand of a finite free $A$-module and hence we see easily that $\omega$ is an epimorphism.

Let $\varphi$ and $\psi$ be $k$-linear mappings of $A$ into itself. The Hochschild coboundary $\delta \varphi$ of $\varphi$ is given by $(\delta \varphi)(a, b)=\varphi(a b)$ $a \varphi(b)-b \varphi(a)$ for $a, b \in A$ (cf. [2]). On the other hand the cupproduct $\varphi-\psi$ of $\varphi$ and $\psi$ is the $k$-bilinear mapping of $A \oplus A$ into $A$ such that $(\varphi-\psi)(a, b)=\varphi(a) \psi(b)$ for $a, b \in A$. Let $P$ and $Q$ be $A$-submodules of $\mathrm{Hom}_{k}(A, A)$, the set of $k$-linear mappings of $A$ into itself. Then the cup-product $P \smile_{Q}$ is the set of $k$-bilinear mappings of $A \oplus A$ into $A$ which are finite sums of mappings of form $\phi \smile \psi$ for $\varphi \in P$ and $\psi \in Q$.

THEOREM 2. Let $A$ be an algebra over a field $k$ such that $\Omega_{k}^{(i)}(A)$ is a finite projective $A$-module for every $i \geqq 1$. Let $\varphi$ be a k-linear mapping of $A$ into iteslf. Then we have $\varphi \in D_{0}^{(q)}(A / k)$ if and only if $\delta \varphi \in D_{0}^{(1)}(A / k) \smile D_{0}^{(q-1)}(A / k)+D_{0}^{(2)}(A / k) \smile D_{0}^{(q-2)}(A / k)+\cdots+D_{0}^{(q-1)}(A / k) \smile$ $D_{0}^{(1)}(A / k)$.

Proof. By Theorem 1 we have an exact sequence

$$
\begin{gathered}
0 \longrightarrow U_{A \otimes A \mid k}^{(q)} \longrightarrow \bigoplus_{i=1}^{q-1} \Omega_{k}^{(i)}(A) \bigotimes_{k} \Omega_{k}^{(q-i)}(A) \\
\stackrel{\Phi_{q}}{\longrightarrow} \bigoplus_{i=1}^{q-2} \Omega_{k}^{(i)}(A) \bigotimes_{k} \Omega_{k}^{(q-1-i)}(A) \longrightarrow 0
\end{gathered}
$$

Our assumption implies that $\Omega_{k}^{(i)}(A) \bigotimes_{k} \Omega_{k}^{(j)}(A)$ is a projective $A \bigotimes_{k} A$ module, and so the above sequence splits. Hence we have an

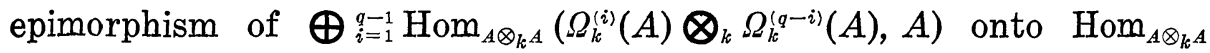
$\left(U_{A \otimes A \mid k}^{\langle q\rangle}, A\right)$, where $A$ is considered as an $A \otimes_{k} A$-module via the contraction mapping: $A \bigotimes_{k} A \rightarrow A$. Since $\Omega_{k}^{(i)}(A)$ is finite $A$-projective, Lemma 4 is applicable to see that $\operatorname{Hom}_{A}\left(\Omega_{k}^{(i)}(A), A\right) \otimes_{k} \operatorname{Hom}_{A}\left(\Omega_{k}^{(j)}(A), A\right)$ is mapped onto $\operatorname{Hom}_{A \otimes_{k} A}\left(\Omega_{k}^{(i)}(A) \otimes_{k} \Omega_{k}^{(j)}(A), A\right)$. Thus we get an epimorphism: $\bigoplus_{i=1}^{q-1} \operatorname{Hom}_{A}\left(\Omega_{k}^{(i)}(A), A\right) \bigotimes_{k} \operatorname{Hom}_{A}\left(\Omega_{k}^{(q-i)}(A), A\right) \rightarrow \operatorname{Hom}_{A \otimes_{k} A}$ $\left(U_{A \otimes \mid(q) k}^{(q)} A\right)$. Let us consider an element $\varphi$ of $D_{0}^{(q)}(A / k)$. The contraction mapping of $A \otimes_{k} A$ into $A$ followed by $\varphi$ is a $q$ th order 
derivation of $A \otimes_{k} A / k$ into $A$. From the direct sum decomposition of $\Omega_{k}^{\langle q)}\left(A \otimes_{k} A\right)$ it follows that $\delta \varphi$ gives an element of $\mathrm{Hom}_{\boldsymbol{A} \otimes_{k} A}$ $\left(U_{A \otimes A / k}^{(q)}, A\right)$. Now only if part is immediate. On the other hand if part is obvious by Proposition 3 of [5].

Remark. The assumption in Theorem 2 is satisfied in the following two cases, and so in these cases Theorem 2 holds.

(1) $A / k$ is a finitely generated field extension.

(2) $A$ is a smooth algebra over a field $k$ ([3] 16.10.1, 16.10.2).

\section{REFERENCES}

1. N. Bourbaki, Éléments de mathématique, Fasc. XXVII. Algèbre commutative. Chap.

I, Actualités Sci. Indust., no. 1290, Hermann, Paris, 1961.

2. H. Cartan and S. Eilenberg, Homological Algebra, Princeton, 1956.

3. A. Grathendieck, Éléments de Géométrie Algébrique IV, Publ. Math. N. 32, 1967.

4. Sandra Z. Keith, High Derivations of Fields, Dissertion, University of Pennsylvania, Philadelphia, Pa., 1971.

5. Y. Nakai, High order derivations I, Osaka J. Math., 7 (1970), 1-27.

Received August 1, 1973.

OSAKA UNIVERSITY 


\section{PACIFIC JOURNAL OF MATHEMATICS}

EDITORS

RICHARD ARens (Managing Editor)

University of California

Los Angeles, California 90024

\section{R. A. Beaumont}

University of Washington

Seattle. Washington 98105

\section{J. DugundjI}

Department of Mathematics University of Southern California Los Angeles, California 90007

D. Gilbarg and J. Milgram

Stanford University

Stanford. California 94305

\section{ASSOCIATE EDITORS}
E. F. BECKENBACH
B. H. NEUMANN
F. WOLF
K. Yoshida

\section{SUPPORTING INSTITUTIONS}

\author{
UNIVERSITY OF BRITISH COLUMBIA \\ CALIFORNIA INSTITUTE OF TECHNOLOGY \\ UNIVERSITY OF CALIFORNIA \\ MONTANA STATE UNIVERSITY \\ UNIVERSITY OF NEVADA \\ NEW MEXICO STATE UNIVERSITY \\ OREGON STATE UNIVERSITY \\ UNIVERSITY OF OREGON \\ OSAKA UNIVERSITY
}

\author{
UNIVERSITY OF SOUTHERN CALIFORNIA \\ STANFORD UNIVERSITY \\ UNIVERSITY OF TOKYO \\ UNIVERSITY OF UTAH \\ WASHINGTON STATE UNIVERSITY \\ UNIVERSITY OF WASHINGTON

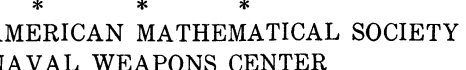

The Supporting Institutions listed above contribute to the cost of publication of this Journal, but they are not owners or publishers and have no responsibility for its content or policies.

Mathematical papers intended for publication in the Pacific Journal of Mathematics should be in typed form or offset-reproduced, (not dittoed), double spaced with large margins. Underline Greek letters in red, German in green, and script in blue. The first paragraph or two must be capable of being used separately as a synopsis of the entire paper. Items of the bibliography should not be cited there unless absolutely necessary, in which case they must be identified by author and Journal, rather than by item number. Manuscripts, in duplicate if possible, may be sent to any one of the four editors. Please classify according to the scheme of Math. Rev. Index to Vol. 39. All other communications to the editors should be addressed to the managing editor, or Elaine Barth, University of California, Los Angeles, California, 90024.

100 reprints are provided free for each article, only if page charges have been substantially paid Additional copies may be obtained at cost in multiples of 50 .

The Pacific of Journal Mathematics is issued monthly as of January 1966. Regular subscription rate: $\$ 72.00$ a year (6 Vols., 12 issues). Special rate: $\$ 36.00$ a year to individual members of supporting institutions.

Subscriptions, orders for back numbers, and changes of address should be sent to Pacific Journal of Mathematics, 103 Highland Boulevard, Berkeley, California, 94708.

\section{PUBLISHED BY PACIFIC JOURNAL OF MATHEMATICS, A NON-PROFIT CORPORATION}

Printed at Kokusai Bunken Insatsusha (International Academic Printing Co., Ltd.), 270, 3-chome Totsuka-cho. Shinjuku-ku, Tokyo 160. Japan.

Copyright (C) 1973 by Pacific Journal of Mathematics Manufactured and first issued in Japan 


\section{Pacific Journal of Mathematics}

\section{Vol. 52, No. $2 \quad$ February, 1974}

Harm Bart, Spectral properties of locally holomorphic vector-valued functions .....

J. Adrian (John) Bondy and Robert Louis Hemminger, Reconstructing infinite

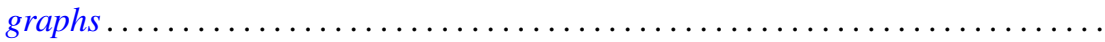

Bryan Edmund Cain and Richard J. Tondra, Biholomorphic approximation of planar

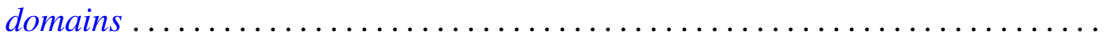

Richard Carey and Joel David Pincus, Eigenvalues of seminormal operators,

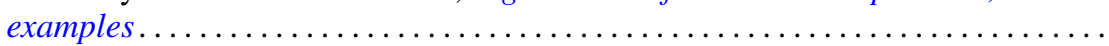

Tyrone Duncan, Absolute continuity for abstract Wiener spaces . . . . . . . . . . . . Joe Wayne Fisher and Louis Halle Rowen, An embedding of semiprime

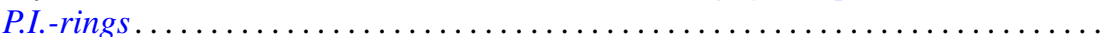

Andrew S. Geue, Precompact and collectively semi-precompact sets of semi-precompact continuous linear operators. . . . . . . . . . . . . . . .

Charles Lemuel Hagopian, Locally homeomorphic $\lambda$ connected plane continua ..... . Darald Joe Hartfiel, A study of convex sets of stochastic matrices induced by

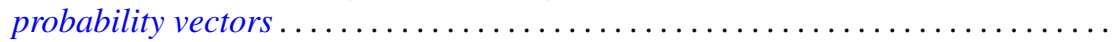

Yasunori Ishibashi, Some remarks on high order derivations $\ldots \ldots \ldots \ldots \ldots \ldots \ldots$ Donald Gordon James, Orthogonal groups of dyadic unimodular quadratic forms.

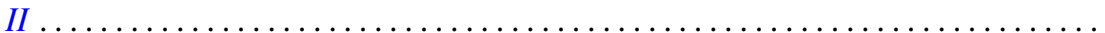

Geoffrey Thomas Jones, Projective pseudo-complemented semilattices . . . . . . . . . Darrell Conley Kent, Kelly Denis McKennon, G. Richardson and M. Schroder,

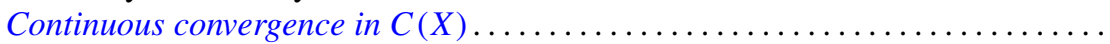

J. J. Koliha, Some convergence theorems in Banach algebras ...

Tsang Hai Kuo, Projections in the spaces of bounded linear oper

George Berry Leeman, Jr., A local estimate for typically real functions . .

475

Andrew Guy Markoe, A characterization of normal analytic spaces by the

homological codimension of the structure sheaf .........

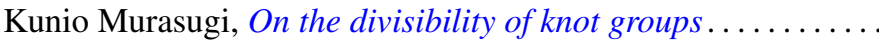

John Phillips, Perturbations of type I von Neumann algebras.

Billy E. Rhoades, Commutants of some quasi-Hausdorff matrices . .

David W. Roeder, Category theory applied to Pontryagin duality

Maxwell Alexander Rosenlicht, The nonminimality of the differential closure .

Peter Michael Rosenthal, On an inversion theorem for the general Mehler-Fock transform pair.

Alan Saleski, Stopping times for Bernoulli automorphisms

John Herman Scheuneman, Fundamental groups of compact complete locally affine complex surfaces. II. ........................

Vashishtha Narayan Singh, Reproducing kernels and operators with a cyclic vector. I. .

Peggy Strait, On the maximum and minimum of partial sums of random variables.

J. L. Brenner, Maximal ideals in the near ring of polynomials modulo 2 .

Ernst Gabor Straus, Remark on the preceding paper: "Ideals in near rings of polynomials over a field" ..........................

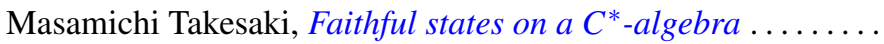

R. Michael Tanner, Some content maximizing properties of the regular simplex.

Andrew Bao-hwa Wang, An analogue of the Paley-Wiener theorem for certain

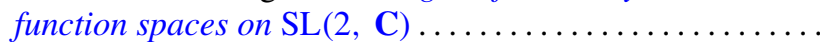

